\title{
Effects of maternal age and stress on offspring quality in a viviparous fly
}

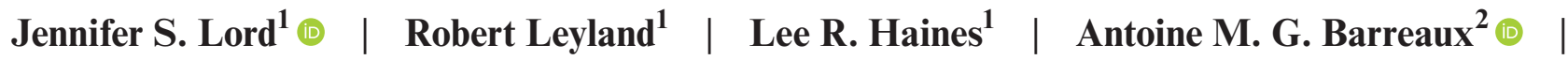 \\ Michael B. Bonsall $^{3}$ | Stephen J. Torr ${ }^{1}$ | Sinead English ${ }^{2}$
}

${ }^{1}$ Department of Vector Biology, Liverpool School of Tropical Medicine, Liverpool,

UK

${ }^{2}$ School of Biological Sciences, University of Bristol, Bristol, UK

${ }^{3}$ Mathematical Ecology Research Group, Department of Zoology, University of

Oxford, Oxford, UK

Correspondence

Jennifer Lord, Department of Vector

Biology, CTID, Liverpool School of

Tropical Medicine, Pembroke Place,

Liverpool, L3 5QA, UK.

Email: jennifer.suzanne.lord@gmail.com

Sinead English, School of Biological Sciences, University of Bristol, Bristol BS8 1TQ.

Email: sinead.english@bristol.ac.uk

Funding information

Biotechnology and Biological Sciences

Research Council, Grant/Award Number:

$\mathrm{BB} / \mathrm{P} 005888 / 1$ and BB/P006159/1

Editor: Jean-Michel Gaillard

\begin{abstract}
Many organisms show signs of deterioration with age in terms of survival and reproduction. We tested whether intraspecific variation in such senescence patterns can be driven by resource availability or reproductive history. We did this by manipulating nutritional stress and age at first reproduction and measuring age-dependent reproductive output in tsetse (Glossina morsitans morsitans), a viviparous fly with high maternal allocation. Across all treatments, offspring weight followed a bell-shaped curve with maternal age. Nutritionally stressed females had a higher probability of abortion and produced offspring with lower starvation tolerance. There was no evidence of an increased rate of reproductive senescence in nutritionally stressed females, or a reduced rate due to delayed mating, as measured by patterns of abortion, offspring weight or offspring starvation tolerance. Therefore, although we found evidence of reproductive senescence in tsetse, our results did not indicate that resource allocation trade-offs or costs of reproduction increase the rate of senescence.
\end{abstract}

K E Y W O R D S

maternal allocation, senescence, tsetse

\section{INTRODUCTION}

Across the natural world, it is commonly observed that as individuals age, they are more likely to die and have lower reproductive output (Hoekstra et al., 2020; Nussey et al., 2013; Zajitschek et al., 2019). Evolutionary theories of senescence are based on the premise that the strength of natural selection declines with age (Hamilton, 1966). Several models have been proposed to explain how such declining selection leads to senescence, based on mutation accumulation (Medawar, 1952) or the pleiotropic effects of mutations (Williams, 1957). Antagonistic pleiotropy occurs when mutations favouring survival or reproduction in early life are at a strong selective advantage, even if these have negative effects on traits in later life (Williams, 1957). An extension of this theory, termed the 'disposable soma' theory, proposes that investment in early-life fitness components, including reproduction, may divert limited resources from somatic maintenance (Kirkwood, 1977). Reproductive senescence could also occur through physiological damage incurred directly from reproduction (McNamara et al., 2009).

There is extensive variation in both the onset and rate of ageing within and across populations and species (Cayuela et al., 2020; Holand et al., 2016; RodríguezMuñoz et al., 2019). Resource allocation trade-offs would

This is an open access article under the terms of the Creative Commons Attribution License, which permits use, distribution and reproduction in any medium, provided the original work is properly cited.

(C) 2021 The Authors. Ecology Letters published by John Wiley \& Sons Ltd. 
be expected to vary by species and context and would explain variation in senescence patterns (Boggs, 2009). An important corollary of these trade-offs is that differences in physiological structures affect evolutionary dynamics and determine the constraints on the evolution of life histories (Clark \& Mangel, 2000; Houston et al., 1988; Mangel \& Clark, 1988). As reproduction is costly (Stearns, 1992), different reproductive histories are likely to affect patterns of reproductive senescence (Boggs, 2009). Scaling these reproductive decisions through physiological drivers provides an appropriate conceptual framework to understand the evolutionary consequences of physiological processes underlying trade-offs (English \& Bonsall, 2019). Focusing on physiological and/or reproductive drivers of fitness allows the variation in senescence patterns to be appropriately quantified. Here, we provide a novel test of these broad ideas by examining the interaction between physiology and reproduction and their effects on life-history patterns of senescence.

We use experimental manipulation of access to mates or food to examine the extent to which variation in senescence is driven by costs of reproduction, allocation of resources or both. For such experiments, insects are useful organisms as they have short generation times, can be reared in large numbers and are easily manipulated. Experiments with Lepidoptera, for example have shown that delayed mating reduces fecundity but extends longevity (Jiménez-Pérez \& Wang, 2009; Unnithan \& Paye, 1991). Female insects with access to fewer resources have also been shown to have lower overall reproductive output but longer lifespan (Chippindale et al., 1993; Ernsting \& Isaaks, 1991; Kaitala, 1991; De Sousza Santos \& Begon, 1987; Tatar \& Carey, 1995).

Experimental studies that have investigated reproductive senescence in insects have yielded mixed results. In many insect species, older mothers produce fewer or smaller offspring (summarised in (Mousseau \& Dingle, 1991)), or with lower survival (Ivimey-Cook \& Moorad, 2020), yet the drivers of intraspecific variation in this senescence remain unclear. In burying beetles Nicrophorus vespilloides, females with experimentally increased reproductive output show faster reproductive ageing (Cotter et al., 2011), whereas in the bedbug Cimex lectularius, aspects of mating (specifically, components of the ejaculate) result in delayed reproductive senescence (Reinhardt et al., 2009). In terms of dietary manipulations, a low-nutrient diet did not affect reproductive senescence in female black field crickets Teleogryllus commodus (Zajitschek et al., 2009), and antler flies Protopiophila litigate show similar age-related decline in mating success when fed on a diet supplemented with protein and sugar (Mautz et al., 2019).

Few studies have focused on reproductive senescence while manipulating both reproduction and nutrition. Here, we present an experimental study, focussing on reproductive senescence in tsetse (Glossina morsitans morsitans), a long-lived Dipteran, under delayed mating and nutritional stress. Tsetse are vectors of human and animal trypanosomiasis in Africa. They give birth to a single larva weighing the same as the mother (Haines et al., 2020; Hargrove \& Muzari, 2015), approximately every 9 days (Hargrove, 2004). Immature stages receive energy and nutrients from the mother only. After birth, the larva buries into the soil and pupates. After $c$. 30 days, an adult emerges. Adults do not increase in size after emergence and female lifespan is approximately 4-12 weeks (Hargrove et al., 2011).

Evidence for age-related changes in reproductive output in laboratory tsetse is mixed (Jordan et al., 1969; Langley \& Clutton-Brock, 1998; McIntyre \& Gooding, 1998). Key limitations to these studies are that flies were kept under optimal laboratory conditions, not kept individually and frequently grouped across ages. Preliminary data from the tsetse colony at the Liverpool School of Tropical Medicine (LSTM) provided evidence of reproductive senescence (Supporting Information, S1 File), but these data did not allow for teasing apart of withinindividual from among-individual patterns (Monaghan et al., 2020). In this study, we therefore used a novel method of collecting data from individual females.

Our first objective was to confirm reproductive senescence in tsetse, as shown by a decline in the number and quality of offspring with maternal age. We also aimed to quantify variability in senescence across individuals, arising from heterogeneity in factors including genetics, size, condition and mate quality. Our second objective was to determine the effect of maternal nutrition and the physiological costs of reproduction on age-dependent patterns of maternal allocation.

\section{MATERIALS AND METHODS}

For the experiments, tsetse from a colony of G. m. morsitans were used (see S2 File for colony details). Colony flies are separated into 12 trays corresponding to each week of life. Each tray holds 12 breeding cages that each house 60 flies. Adults in the colony older than 84 days are killed due to a decline in reproductive output. To select females for the experiment, we collected equal numbers of pupae from each tray over a 1-week period (S2 File). On emergence, we randomly assigned 96 females to one of three experimental treatments: control, nutritional stress or mating delay (Figure 1, S2 File).

\section{Experimental manipulation of female nutrition and age at first breeding}

We diluted red blood cells with serum to produce a lowquality diet for the nutritional stress treatment. Reducing the amount of haemoglobin results in lower pupal wet weights (Kabayo \& Langley, 1985). Trials testing different ratios of red blood cells to serum showed that flies 
GROUPS

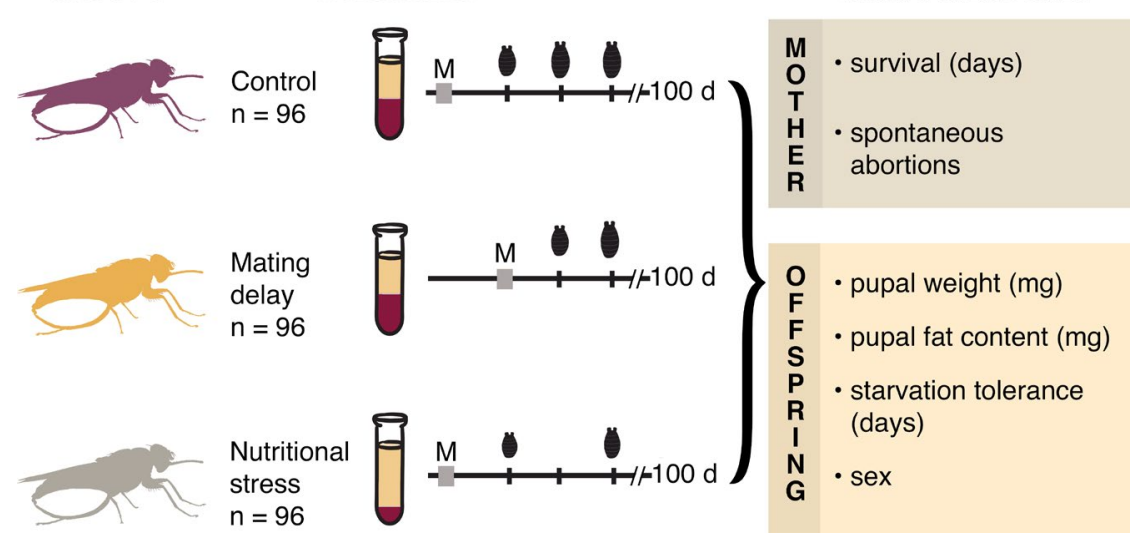

F I G U R E 1 Overview of experiments. M-mating. Colour-coded silhouette of pregnant tsetse with larva, timeline shows a silhouette of a pupa and tubes containing blood, the dark red indicates red blood cells and beige indicates serum. The first pupae produced by the female tsetse is always smaller than the offspring she produces during subsequent reproductive cycles. Our data show that the nutritionally stressed group produces smaller pupae and as expected, due to fertilisation delay, the mating delayed group does not produce as many pupae during the experimental time course. The nutritionally stressed group also shows two pupae with a gap in between to depict the overall reduction in pupal numbers and the higher chance larvae is not birthed during every 10 -day reproductive cycle

fed on c. $10 \%$ red blood cells produced lighter pupae but had similar survival over a 50-day period, compared to flies fed on blood used to feed the colony with c. $45 \%$ red blood cells (S3 File). We thus ascribed 10\% red blood cells as the 'nutritional stress' treatment and $45 \%$ red blood cells as 'control'.

Females in the control and nutritional stress treatments were mated at emergence. For delayed mating, virgin females were kept in communal cages for 3 weeks post-emergence. Virgin females continue to ovulate, but mature eggs disintegrate and are re-absorbed (Ejezie \& Davey, 1977). At the time of mating, females in all treatments were mated for $48 \mathrm{~h}$ at a ratio of two females to one, 1-week-old male. Although most females likely mated within this time, it was not possible to determine successful mating.

Once mated, females were placed into individual cages (described in S2 File), to allow recording of individual female reproductive output and survival. Cameras were fixed below cages to record weekend activity and to identify exact larviposition dates. Females were monitored daily between Monday and Friday and the date of any deaths recorded. To determine the exact date of death for females found dead on a Monday morning, we reviewed video recordings from the previous weekend (S2 File).

Experimental flies were fed following the colony schedule (S2 File). The experiment was run until mothers were 100 days old. The probability of survival for females up to 100 days is estimated to be c. $10 \%$ in the wild (Hargrove et al., 2011). In addition, unpublished data from the LSTM colony indicated that by 84 days the probability of producing a dead larva-considered as a spontaneous abortion ('abortion' hereafter) - had increased from c. 0 to 0.25 . The size of pupae produced had also declined to similar sizes seen from first-time mothers, after a peak at 7 weeks of age (S1 File). Finally, for stationary populations in nature, the age-related strength of selection against mortality or for fertility is approximately proportional to rates of cumulative survival, predicting that selection for these traits has relaxed by roughly $90 \%$ by 100 days compared to early in life (Hamilton, 1966). For these reasons, we deemed a duration of 100 days to be sufficient to quantify age-related changes in reproductive output.

\section{Offspring measurements}

Time of larviposition was recorded and production of a dead larva was considered an abortion (Baldry et al. 1992). It was not possible to record egg abortions. For pupae collected on a Monday morning, we consulted video recordings to determine the exact day of larviposition (S2 File). For aborted larvae, however, we could not determine the date for those aborted over the weekend because early larval stages were not visible to the camera and were therefore recorded as occurring on Friday. For pupae, we measured the wet weight, to $0.1 \mathrm{mg}$. Wet weight is a non-destructive method of fly size, and field studies have shown that it increases by $0.82 \mathrm{mg}$ for each $1 \mathrm{~mm}^{3}$ increase in pupal volume (Hargrove, 1999). Of the pupae produced, we randomly selected $70 \%$ of samples to record emergence. The remaining 30\% were destructively sampled for fat analysis (S2 File) to quantify how wet weight correlates with fat reserves.

We recorded emergence because the probability of emergence is dependent on fat reserves (Phelps, 1973). Pupae assigned for emergence were placed singly into $50 \mathrm{ml}$ Falcon tubes with a $3 \mathrm{~mm}$ hole in the cap to allow airflow. Pupae were observed daily for emergence on working days and again video recordings were used to determine the date of emergence on weekends. Pupae 
were observed for a maximum of 50 days. The average pupal duration at $26^{\circ} \mathrm{C}$ is c. 25 days, so any pupae that were going to emerge should have by 50 days (Phelps \& Burrows, 1969). Any pupae that had not emerged were recorded as a failed emergence (Hargrove, 2004).

Each emerging fly remained in the tube until it died of starvation. We measured starvation tolerance because, in the wild, newly emerged tsetse have a high probability of mortality (Hargrove et al., 2011), likely due to failure to find a bloodmeal before running out of fat reserves. Indeed, evidence of an increase in mortality for smaller flies, occurring at emergence, has been recorded in the field (Dransfield et al., 1989). Sex of emerging flies and date of death were recorded.

From preliminary data (S1 File), we hypothesised that there would be reproductive senescence across all treatments in the form of increased probability of abortion, decreased offspring wet weight and lower starvation tolerance with advancing maternal age. If resource allocation trade-offs drive variation in senescence, we expected nutritionally stressed females to exhibit a more rapid decline in reproductive output with age, as they have relatively fewer resources for somatic maintenance. However, if physiological costs of reproduction drive variation in senescence, then stressed females-with lower reproductive output - may incur less physiological damage, and thus have slower senescence than controls. Females with delayed mating are predicted to have slower reproductive senescence both due to allocation tradeoffs (more resources to allocate to somatic maintenance) and costs of reproduction (which have been alleviated in the early stage of life). Lastly, we hypothesised that the effect of maternal age on starvation tolerance would be determined largely by energy reserves, as indirectly measured by offspring wet weight. Heavier offspring would have more reserves and therefore have higher starvation tolerance.

\section{Statistical analyses}

For each analysis, models were compared using Akaike's information criterion (AIC). If the ratio of the sample size to the number of model parameters was $<40$, we used AIC corrected for small sample size (AICc) (Burnham $\&$ Anderson, 2002). Figures were produced using the model with the lowest AIC. For model comparison, the difference in AIC between each model and the lowest AIC model $\left(\Delta_{i}\right)$ and Akaike weights $\left(\omega_{i}\right)$ was calculated. Akaike weights sum to one and provide a relative indication for the weight of evidence for any one model as the best approximating model (Burnham \& Anderson, 2002). We analysed the data using $R$ version 3.6 ( $R$ Core Team, 2014). The data and $\mathrm{R}$ scripts can be accessed at (https://github.com/jenniesuz/tsetse_senescence.git).

We carried out statistical analyses for each treatment separately using linear and generalised linear mixed effects models implemented with the 'Ime4' and 'nlme' R packages (Bates et al., 2015; Pinheiro et al. 2018). We did not include treatment as an interaction term in models, as the mating delay and nutritional stress treatment produced different phenomena and were therefore not directly comparable within the same model. This was reflected in the differences in variance structure between the treatments. To test for differences between treatments of the effect of maternal age on the probability of abortion and offspring wet weight, parameter estimates from models fitted to data from the nutritional stress and mating delay treatments were compared with those from the control using a z-test.

Maternal age in days was incorporated as a continuous variable in analyses. Maternal age at last observation was also included as a continuous covariate in models of offspring wet weight and starvation tolerance. This was to account for possible linear effects of removal of mothers that died before the end of the experiment on model fits and coefficients (Van De Pol \& Verhulst, 2006). Age at last observation was either the age of death or the age at the end of the experiment. We did not, however, account for age at last observation in models of the probability of abortion (this is explained in S4 File).

All models with and without random effects were fitted and simultaneously compared using maximum likelihood estimation, assuming that the bias in the variance components would be relatively small $(n /(n-p): 1.009$ for the probability of abortion; 1.049 for offspring wet weight; and 1.095 for offspring survival, largest values across treatments reported). Models with and without a random intercept were compared to assess variation among individual mothers in the probability of abortion, offspring wet weight and offspring starvation tolerance. We also compared models with and without a random slope for maternal age, to assess variation in senescence patterns among individual mothers.

Logistic regression was used to quantify the effect of maternal age on the probability of abortion. For models of offspring wet weight and starvation tolerance, we compared the effects of maternal age as a quadratic, logarithmic or linear effect. For wet weight, we also compared the most parsimonious model with a model fit using generalised additive modelling (GAM). Cubic regression splines were fitted for each treatment, with maternal age as the explanatory variable and accounting for multiple offspring from individual mothers, using the 'mgcv' $\mathrm{R}$ package (Wood, 2017). Generalised additive model fits with knots-locations where the slope changes-ranging from 3 to 10 were compared using AICc. The correlation between offspring wet weight and fat was summarised using Pearson's correlation coefficient.

For offspring starvation tolerance, there was no censoring, as all emerged flies were dead by the end of the experiment, and the data were approximately normally distributed (Fig. S5). The relationship between offspring wet weight, sex, maternal age and number of days to 
starvation was therefore modelled using linear mixed effects models. Maternal age was included in models as described for offspring wet weight. In addition to maternal age and wet weight, we also included offspring sex as females are larger than males on emergence (Hargrove et al., 2019). For both wet weight and starvation tolerance, we report conditional and marginal $R^{2}$ (Nakagawa \& Schielzeth, 2013). Lastly, an analysis of maternal survival is provided in S6 File.

\section{RESULTS}

\section{Abortions}

There was strong evidence for an effect of age on the probability of abortion (models without age: control $\omega=0.000$; mating delay $\omega=0.001$; and nutritional stress $\omega=0.000$ ) (Tables S7 1-3). The odds of abortion increased with age across treatments (odds from model with lowest AIC: control 1.072, C.I. 1.054-1.094; mating delay 1.053, CI 1.025-1.086; and nutritional stress 1.047, CI 1.034-1.061) (Figure 2, S7 4-6). The estimated increase in odds of abortion with age was not significantly different between the mating delay and control treatments $(z=1.03, p=0.15)$, but was significantly lower in the nutritional stress treatment, relative to the control $(z=2.10, p=0.02)$. This was the case even when maternal age at last observation was included as a covariate in the nutritional stress model (S4 File). However, females in the nutritional stress treatment had a higher probability of abortion at any age compared with the control (Figure 2).

For all treatments, including both a random intercept and slope in models resulted in a singular fit indicating that models were overfitted. There was, however, strong evidence for variation among mothers in the probability of abortion for the nutritional stress treatment (model with random intercept $\omega=0.985$; model without $\omega=0.015$ ). This was not the case for the control (model with random intercept $\omega=0.289$; model without $\omega=0.711$ ) or mating delay treatments (model with random intercept $\omega=0.368$; model without $\omega=0.631$ ) (Tables S7 1-3).

\section{Offspring wet weight and fat}

Across all treatments, wet weight of pupae increased and then declined with maternal age (Figure 3). There was

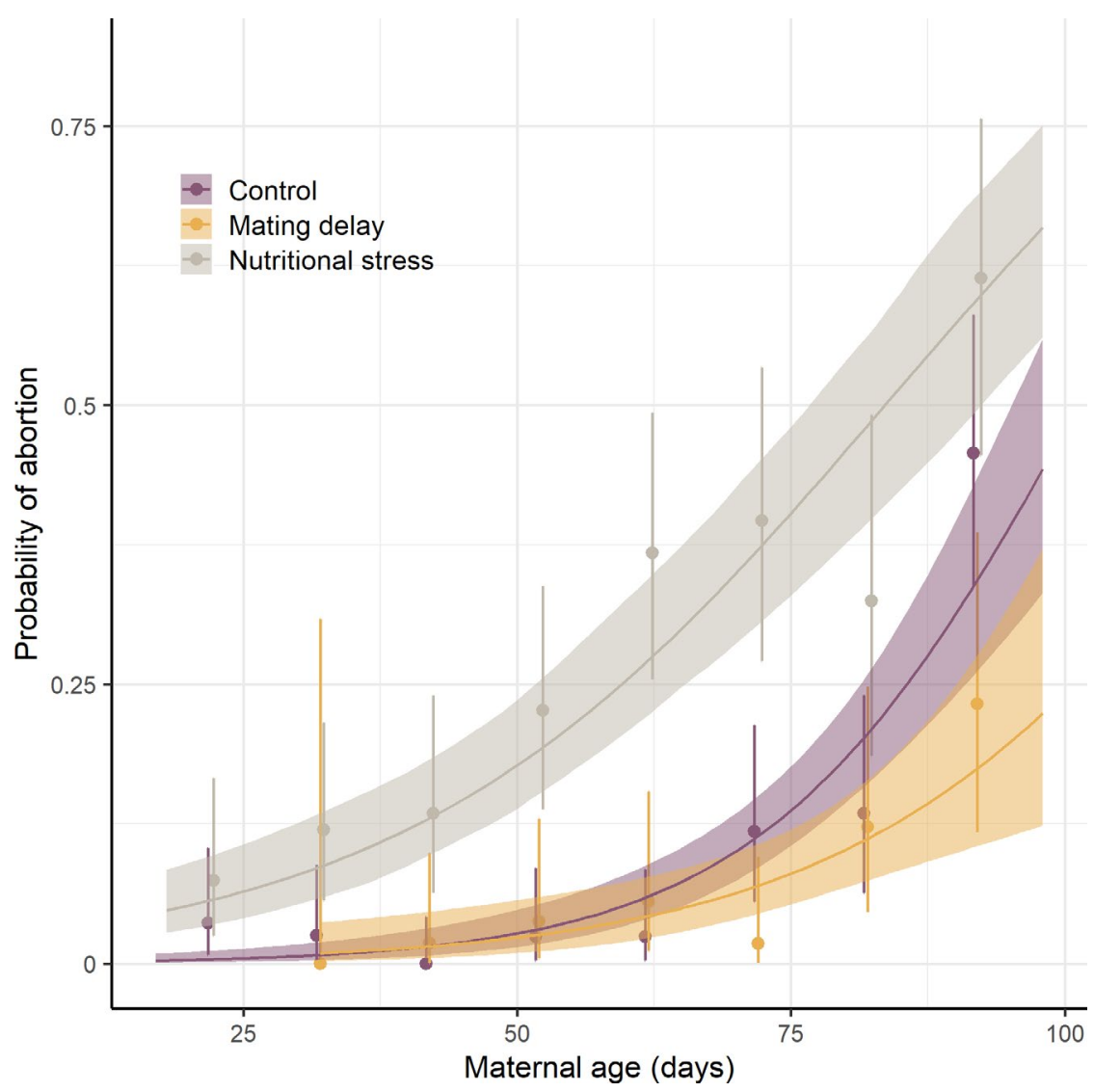

F IGURE 2 Predicted probability of larval abortion as a function of maternal age, by treatment. Predicted probabilities from generalised linear mixed effects model fits to the data and $95 \%$ prediction intervals. Points-mean for 10 -day intervals and exact binomial $95 \%$ confidence intervals. Plots of raw data are provided in S10 File 


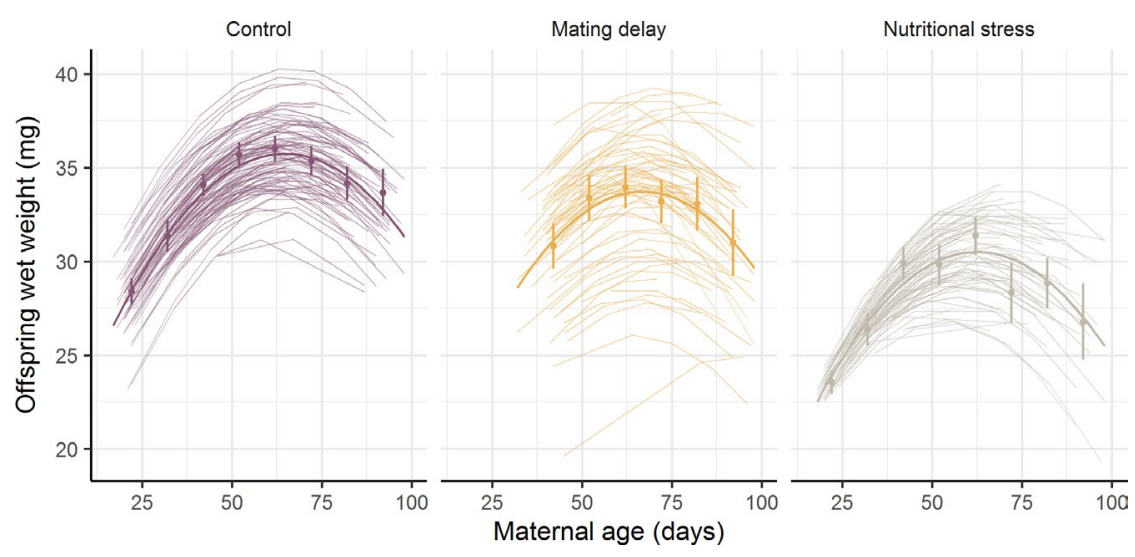

F I G U R E 3 Offspring wet weight as a function of maternal age and treatment. Showing model fits to the data: thick line-population level, thinner lines-individual level. Points - average wet weights for 10-day intervals and $95 \%$ confidence intervals. Plots of raw data are provided in S10 File

strong evidence for a quadratic effect of maternal age on offspring wet weight; for all treatments, Akaike weights for models including a quadratic effect summed to one (Tables S7 7-9). In addition, across treatments, the predicted effect of maternal age at the population level, assuming a quadratic fit, was within the standard errors of predictions from GAM fits to the data (S8 File). The fitted quadratic curves were qualitatively similar across treatments. Fitted model coefficients for maternal age (Tables S7 10-12) from the nutritional stress and mating delay treatments were not significantly different from that of the control (nutritional stress: $z=0.27, p=0.39$; mating delay: age $z=-0.55, p=0.30$ ). The coefficients for maternal age squared were also not significantly different (nutritional stress: $z=0.05, p=0.48$; mating delay: $z=0.33, p=0.38$ ). The conditional $\mathrm{R}^{2}$ was also similar across treatments: 0.640 for the control; 0.658 for the mating delay; and 0.526 for the nutritional stress treatment. However, the marginal $\mathrm{R}^{2}$ was lower for the mating delay treatment: 0.071 compared with 0.379 for the control; and 0.291 for nutritional stress. This was also reflected in the random effects variance as described below.

For all three treatments, there was strong evidence for individual variation among mothers in the wet weight of their pupae (for all treatments, all models without random effects $\omega=0.000$ ). This variation was highest in the mating delay treatment with a random intercept variance from the most parsimonious model of 11.700 , compared with 4.695 for the control and 2.331 for the nutritional stress treatment (Tables S7 13-15). There was evidence for variation among mothers in the effect of maternal age on wet weight for the control and nutritional stress treatments (quadratic fit with random intercept and slope: control $\omega=0.807$; and nutritional stress $\omega=0.958$ ). There was less support for such age-related variation in the mating delay treatment (quadratic fit with random intercept and slope $\omega=0.659$ ). Offspring fat increased linearly with offspring wet weight (Pearson's correlation coefficient $0.554, n=364$ ) (File S9), thus for brevity, we focussed only on wet weight as our trait of interest.

\section{Offspring emergence and starvation tolerance}

During the observation period, $96 \%$ of 355 pupae from the control, $93 \%$ of 187 pupae from the mating delay and $91 \%$ of 232 pupae from the nutritional stress groups emerged successfully. All emerged offspring subjected to starvation were dead by 15 days' post-emergence. Although the wet weight of pupae was similar between females and males (Figure 4a), there was strong evidence that female offspring survived longer than males, but not by more than a day (Tables S7 19-21), with $\omega \leq 0.01$ for models not including sex (Tables S7 16-18, Figure 4b).

There was strong evidence for an additional effect of maternal age on offspring starvation tolerance, in addition to offspring wet weight, for the nutritional stress treatment (models without age $\omega=0.000$ ). Offspring from young mothers in the nutritional stress treatment were particularly vulnerable to starvation (Figure $4 b$, quadratic effect of maternal age including random intercept $\omega=0.868$ ). While there was also evidence for maternal age effects on offspring starvation tolerance in the other two treatments, there was not sufficient information in the data to quantify the relationship between maternal age and offspring starvation tolerance (lowest AIC model, control: quadratic effect and no random effects $\omega=0.379$; mating delay: quadratic effect with no random effects $\omega=0.276$ ) (Tables S7 $16-17)$. For the lowest AIC models, the marginal $R^{2}$ was 0.337 for the control, 0.191 for the mating delay and 0.551 for the nutritional stress treatments. The conditional $R^{2}$ was $0.347,0.291$ and 0.634 respectively.

For the mating delay treatment, singular fits were obtained for models assuming a quadratic effect of maternal age and including a random intercept and slope. There was also insufficient information in the data to provide evidence for among-mother variation in the control treatment, with the difference in AIC weights between models with and without random effects $<0.18$ (Tables S7 16-18). However, there was evidence for an effect of individual mother on starvation tolerance in the nutritional stress treatment (all models without random 
(a)

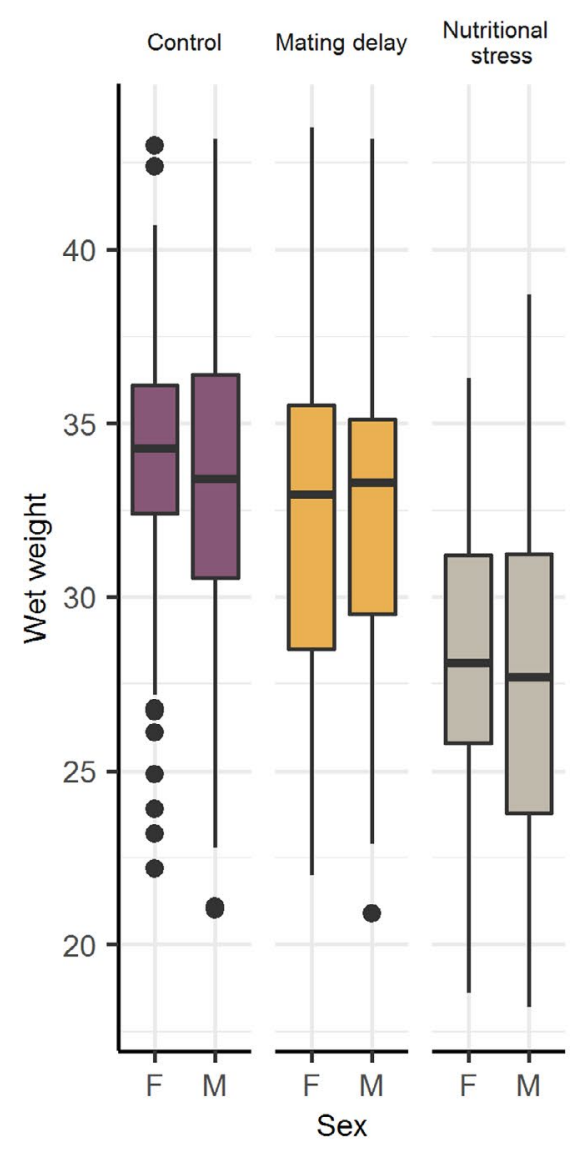

(b)
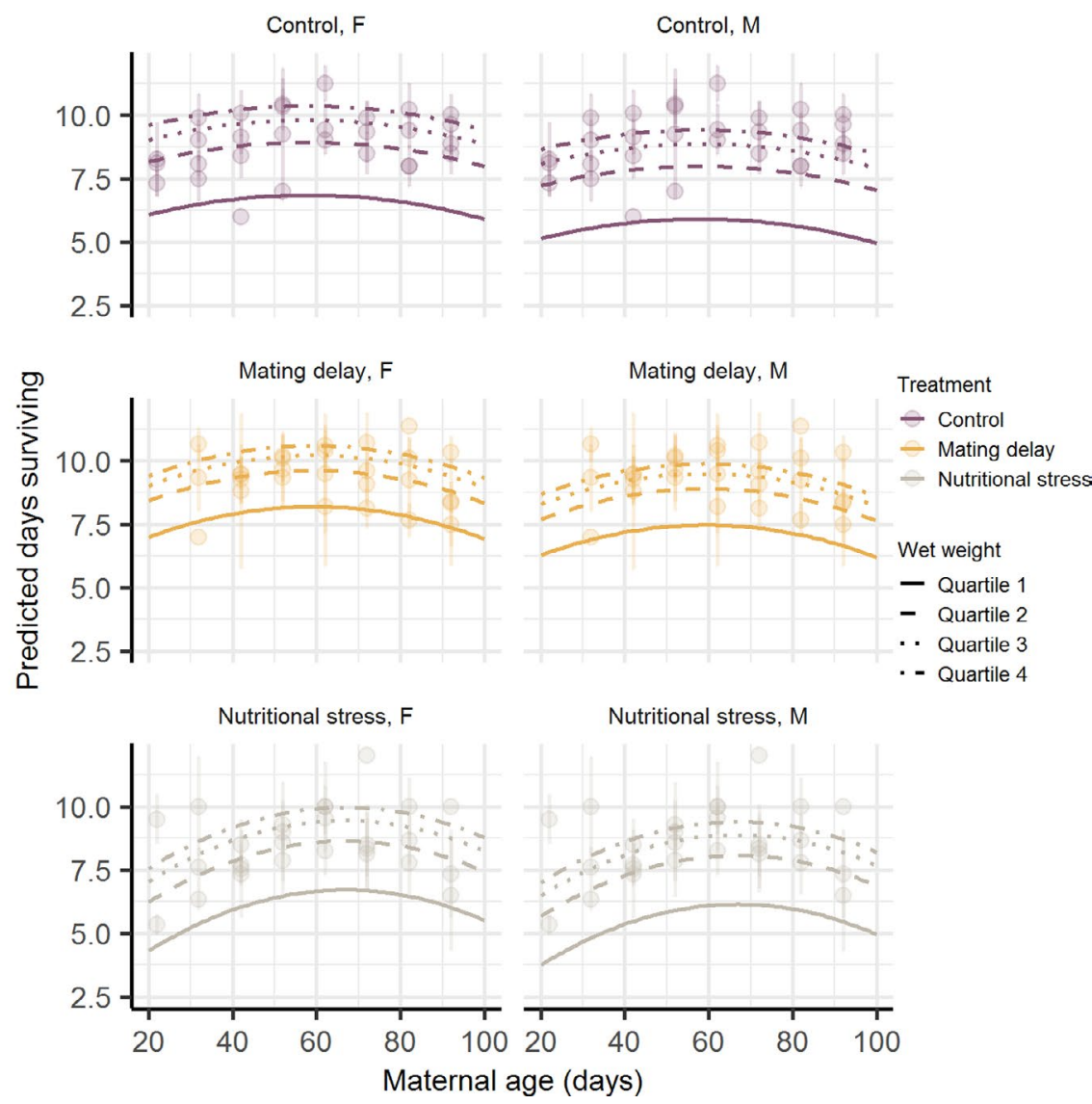

F I G U R E 4 Effect of sex, wet weight and maternal age on starvation tolerance (the number of days a newly emerged fly can survive starvation). (a) Wet weight as a function of offspring sex by treatment; (b) Predicted survival time based on linear mixed effects model. Days adults survived starvation is plotted against maternal age. Prediction for each wet weight quartile shown. Points-average wet weights for 10day intervals and 95\% confidence intervals. Plots of raw data are provided in S10 File

effects $\omega \leq 0.09$ ) (Tables S7 16-18), but not for variation among individual mothers in the effect of maternal age on offspring starvation tolerance $(\omega \leq 0.022)$.

\section{DISCUSSION}

While there is evidence of actuarial and reproductive senescence in insects (Nussey et al., 2013; Zajitschek et al., 2019), the drivers of variation in senescence patterns are not fully understood. Theory suggests that physiological factors influence the expected variation in life-history patterns such as senescence (Boggs, 2009; English \& Bonsall, 2019). Here, we manipulated both nutrition and age at mating to quantify reproductive senescence in tsetse; a viviparous fly with high maternal allocation and iteroparous reproduction. Both offspring weight and starvation tolerance declined with maternal age, after a peak, yet these patterns of senescence were similar across treatments. We did not find that manipulating access to nutrition or the physiological costs of reproduction changed the onset or rate of reproductive senescence, in terms of offspring quality. However, nutritional stress increased the abortion rate, reduced the size of offspring and reduced survival, especially of offspring born to younger mothers. Unexpectedly, although the probability of abortion was higher in the nutritional stress treatment at any age, the increase in the odds of abortion with age was lower for this treatment relative to the control.

The bell-shaped relationship of offspring quality with maternal age may have contributed to the relatively small effects of age evident in previous studies where grouped ages and mean values were used (Langley \& Clutton-Brock, 1998; McIntyre \& Gooding, 1998). The bell-shaped pattern was similar across treatments, and reflects the general pattern of reproductive output, or offspring quality, observed across diverse taxonomic groups for iteroparous species (e.g. Sharp \& CluttonBrock, 2010; Velando et al., 2006). As summarised by Monaghan et al. (2020), this pattern can arise from population-level effects, due to selective disappearance, or individual-level effects. By tracking individual mothers and including maternal age at last observation as a covariate in models, we provide evidence that, for tsetse, the bell-shaped relationship between offspring wet weight and maternal age is a consequence of individual 
effects. The existence of such bell-shaped curves could be explained by natural selection, where the strength of selection for an age-specific maternal effect on offspring size and early survival is relatively weak in young mothers, strengthens as they age and then weakens again (Moorad \& Nussey, 2016). For tsetse, the initial increase in offspring wet weight could also be a consequence of newly emerged females having to divert resources away from reproduction for the development of thoracic muscles (Anderson \& Finlayson, 1973).

Tracking individual mothers provided insights into individual variation in maternal allocation, as is highlighted by long-term studies in wild vertebrate populations (Douhard et al., 2020; Froy et al., 2013; Hamel et al., 2012). There was marked variation in offspring wet weight among mothers, particularly for the mating delay treatment, and variation in senescence patterns for wet weight, particularly for nutritionally stressed mothers. Some females in the mating delay treatment consistently produced smaller than average offspring and these females contributed more to the individual heterogeneity than those producing consistently heavier offspring. For nutritionally stressed mothers, variation between individuals in offspring wet weight increased as mothers aged. These observations suggest that variation in offspring quality is affected not only by mother size but also unmeasured aspects of her condition. The large amount of variation between individuals in offspring weight and changes in the extent of variation with maternal age in this study, was unexpected. Future studies quantifying the relative roles of mother size and physiological condition on offspring size across different ages would be valuable.

Offspring from young mothers that were nutritionally stressed had the lowest starvation tolerance. Maternal age also affected offspring starvation tolerance independently of age-effects mediated through wet weight, suggesting that there may be other factors associated with age that influence the quality of offspring. More subtle effects of maternal age on the quality of resources transferred to offspring warrant further investigation. For tsetse, during the late stages of pregnancy, females transfer fat and amino acids (Langley 1977). It may be that young, nutritionally stressed females are limited in crucial amino acids. High amounts of tyrosine in the gut contents of third instar larvae, which is involved in the tanning of larval and adult cuticles have been observed (Cmelik et al., 1969). The authors reasoned that the tyrosine and phenylalanine obtained from a single bloodmeal is unlikely to be sufficient to meet the amount required by offspring and that a surplus stored from previous bloodmeals may be necessary. Size may be limited when specific nutrients are limited, irrespective of the amount of energy available. Resource allocation processes are also likely more complex, and more nuanced studies on the effects of the quality of resources as well as quantity may be required to understand the ageing process. Indeed, maternal effects studies for other insects have shown that quality of resources as well as their quantity influences offspring fitness traits (Kyneb \& Toft, 2006; Wen et al., 2020).

We found that females experiencing nutritional stress have a relatively high rate of abortion, in line with studies of general stress-associated abortions in other viviparous species, including meerkats (social stress) (Young et al., 2006) and sharks and rays (capture-induced stress) (Adams et al., 2018). In tsetse, the dynamics of nutritional allocation during pregnancy may favour a strategy of earlier abortions in adverse conditions. Hargrove and Muzari (2015), using field-collected G. pallidipes, showed that the majority of fat is transferred to the larva only after c. $80 \%$ pregnancy has been completed. Therefore, a female could potentially abort a larva if there are not enough fat reserves for a full-term pregnancy.

While the probability of abortion was higher in the nutritional stress treatment at any age, the increase in the odds of abortion with age was lower for the nutritional stress treatment relative to the control. Although the difference is small, it results in important cumulative differences over time (see the slopes in Figure 2 after c. 50 days of age). Baseline levels of abortion were higher in the nutritional stress treatment. For the control treatment, the probability of abortions increased (non-additively) beyond maternal ages of c. 50 days, suggestive of further constraints on reproductive senescence. These results remain intriguing and warrant further investigation.

We focused on reproductive senescence in this study. One limitation is that we did not continue the experiment to quantify maternal survival fully. Our evidence of actuarial senescence in the nutritional stress treatment supports analyses of mark-recapture studies of $G$. $m$. morsitans in the field (Hargrove et al., 2011). An additional experiment to test whether late-mated females experience a delayed onset in actuarial senescence would be informative, given the similar rates of reproductive senescence observed across treatments. The findings from this experiment could also then be compared with those from other insect studies focusing on actuarial senescence (Jiménez-Pérez \& Wang, 2009; Unnithan \& Paye, 1991). Our conclusions with respect to reproductive senescence are also constrained to this 100-day period. While, in principle, it would be optimal to run the experiment until all females had died, the $>1 / 4$ of offspring being aborted and fewer females surviving would result in relatively small sample sizes beyond this time point. Lastly, we acknowledge that our conclusions are based on a laboratory study, and thus we cannot rule out effects of inbreeding or inadvertent selection. Further study of tsetse in the field would need to be carried out to confirm whether such age-dependent patterns of reproductive output extend to wild populations.

To conclude, our results provide evidence of a bellshaped relationship between maternal age and offspring quality at the individual-level for the iteroparous tsetse fly. This complements the existing body of work from other species, which has predominantly shown a similar curve for the 
number of offspring produced as mothers age (Monaghan et al., 2020). Contrary to predictions based on resource allocation trade-offs or damage accumulation, however, our study did not find evidence of an earlier or more rapid decline in offspring quality with maternal age as a function of direct costs of reproduction or reduced resources.

\section{ACKNOWLEDGEMENTS}

We thank Jessica Lingley for assistance in the analysis of the fat content of pupae and Glyn Vale for input on an earlier version of the manuscript, and John Hargrove, Matt Keeling and Kat Rock for general discussion. We thank Daniel May for digitising the LSTM tsetse colony laboratory books which provided important pilot data for our experiments. The research was funded by the Biotechnology and Biological Sciences Research Council, grant numbers BB/P005888/1 and BB/P006159/1. SE was supported by a Royal Society Dorothy Hodgkin Fellowship (DH140236).

\section{AUTHORSHIP}

SE and LRH designed the study with inputs from SJT, JSL and MBB. RL and LRH carried out the laboratory experiments. JSL carried out the analyses with input from SE, MBB and SJT and led the writing of the manuscript. RL, LRH, AMGB, MBB, SJT and SE contributed to manuscript content and structure.

\section{PEER REVIEW}

The peer review history for this article is available at https://publons.com/publon/10.1111/ele.13839.

\section{DATA AVAILABILITY STATEMENT}

The data and $\mathrm{R}$ scripts to reproduce the analyses will be available at https://github.com/jenniesuz/tsetse_senes cence.git.

\section{ORCID}

Jennifer S. Lord (10 https://orcid.org/0000-0001-6616-1526

Antoine M. G. Barreaux (10 https://orcid.

org/0000-0001-5822-761X

Sinead English (10) https://orcid.org/0000-0003-2898-2301

\section{REFERENCES}

Adams, K.R., Fetterplace, L.C., Davis, A.R., Taylor, M.D. \& Knott, N.A (2018) Sharks, rays and abortion: The prevalence of capture-induced parturition in elasmobranchs. Biological Conservation, 217, 11-27.

Anderson, M. \& Finlayson, L.H. (1973) Ultrastructural changes during growth of the flight muscles in the adult tsetse fly, Glossina austeni. Journal of Insect Physiology, 19, 1989-1997.

Baldry, D., Boreham, P., Challier, A., Van Etten, J., Everts, J., Gravel, J., et al. (1992). Training manual for tsetse control personnel Volume 1. Food and Agriculture Organization of the United Nations.

Bates, D., Maechler, M., Bolker, B. \& Walker, S. (2015) Fitting linear mixed-effects models using lme4. Journal of Statistical Software, $67,1-48$.

Boggs, C.L. (2009) Understanding insect life histories and senescence thorugh a resource allocation lens. Functional Ecology, 23, 27-37.

Burnham, K. \& Anderson, D. (2002) Model selection and multimodel inference: a practical information-theoretic approach., 2nd edn. New York Inc: Springer-Verlag.
Cayuela, H., Lemaître, J.F., Bonnaire, E., Pichenot, J. \& Schmidt, B.R. (2020) Population position along the fast-slow life-history continuum predicts intraspecific variation in actuarial senescence. Journal of Animal Ecology, 89(4), 1069-1079.

Chippindale, A.K., Leroi, A.M., Kim, S.B. \& Rose, M.R. (1993) Phenotypic plasticity and selection in Drosophila life-history evolution. I. Nutrition and the cost of reproduction. Journal of Evolutionary Biology, 6, 171-193.

Clark, C. \& Mangel, M. (2000) Dynamic state variable models in ecology. Oxford, UK: Oxford University Press.

Cmelik, S.H.W., Bursell, E. \& Slack, E. (1969) Composition of the gut contents of third-instar tsetse larvae (Glossina morsitans westwood). Comparative Biochemistry and Physiology, 29, 447-453.

Cotter, S.C., Ward, R.J.S. \& Kilner, R.M. (2011) Age-specific reproductive investment in female burying beetles: Independent effects of state and risk of death. Functional Ecology, 25, 652-660.

De Sousza Santos, P. \& Begon, M. (1987) Survival costs of reproduction in grasshoppers. Functional Ecology, 1, 215-221.

Douhard, M., Festa-Bianchet, M. \& Pelletier, F. (2020) Sons accelerate maternal aging in a wild mammal. Proceedings of the National Academy of Sciences, 117, 4850-4857.

Dransfield, R.d., Brightwell, R., Kiilu, J., Chaudhury, M.F. \& Abie, D.A.A. (1989) Size and mortality rates of Glossina pallidipes in the semi-arid zone of southwestern Kenya. Medical and Veterinary Entomology, 3, 83-95.

Ejezie, G.C. \& Davey, K.G. (1977) Some effects of mating in female tsetse, Glossina austeni newst. Journal of Experimental Zoology, 200, 303-310.

English, S. \& Bonsall, M.B. (2019) Physiological dynamics, reproduction-maintenance allocations, and life history evolution. Ecology and Evolution, 9, 9312-9323.

Ernsting, G. \& Isaaks, J.A. (1991) Accelerated ageing: A cost of reproduction in the carabid beetle notiophilus biguttatus F. Functional Ecology, 5, 299.

Froy, H., Phillips, R.A., Wood, A.G., Nussey, D.H. \& Lewis, S. (2013) Age-related variation in reproductive traits in the wandering albatross: Evidence for terminal improvement following senescence. Ecology Letters, 16, 642-649

Haines, L.R., Vale, G.A., Barreaux, A.M.G., Ellstrand, N.C., Hargrove, J.W. \& English, S. (2020) Big baby, little mother: Tsetse flies are exceptions to the juvenile small size principle. BioEssays, 42(11), 2000049

Hamel, S., Craine, J.M. \& Towne, E.G. (2012) Maternal allocation in bison: Co-occurrence of senescence, cost of reproduction, and individual quality. Ecological Applications, 22, 1628-1639.

Hamilton, W. (1966) The moulding of senescence by natural selection. Journal of Theoretical Biology, 12, 12-45.

Hargrove, J. (1999) Nutritional levels of female tsetse Glossina pallidipes from artificial refuges. Medical and Veterinary Entomology, 13, 150-164.

Hargrove, J. (2004). Tsetse population dynamics. In: Maudlin, I., Holmes, P. \& Miles, M. (Eds.) The trypanosomiases. Wallingford, UK: CABI Publishing, pp. 113-135.

Hargrove, J., English, S., Torr, S.J., Lord, J., Haines, L.R., van Schalkwyk, C. et al. (2019) Wing length and host location in tsetse (Glossina spp.): Implications for control using stationary baits. Parasites and Vectors, 12, 1-13.

Hargrove, J. \& Muzari, M. (2015) Nutritional levels of pregnant and postpartum tsetse Glossina pallidipes Austen captured in artificial warthog burrows in the Zambezi Valley of Zimbabwe. Physiological Entomology, 40, 138-148.

Hargrove, J., Ouifki, R. \& Ameh, J. (2011) A general model for mortality in adult tsetse (Glossina spp.). Medical and Veterinary Entomology, 25, 385-394.

Hoekstra, L.A., Schwartz, T.S., Sparkman, A.M., Miller, D.A.W. \& Bronikowski, A.M. (2020) The untapped potential of reptile biodiversity for understanding how and why animals age. Functional Ecology, 34(1), 38-54. 
Holand, H., Kvalnes, T., Gamelon, M., Tufto, J., Jensen, H., Pärn, H. et al. (2016) Spatial variation in senescence rates in a bird metapopulation. Oecologia, 181, 865-871.

Houston, A.I., Colin, C., John, M. \& Marc, M. (1988) Dynamic models in behavioural and evolutionary ecology. Nature, 332, 29-34.

Ivimey-Cook, E. \& Moorad, J. (2020) The diversity of maternal-age effects upon pre-adult survival across animal species: Diversity of maternal-age effects. Proceedings of the Royal Society, 287, 20200972.

Jiménez-Pérez, A. \& Wang, Q. (2009) Effect of mating delay on the reproductive performance of Cnephasia jactatana (Lepidoptera: Tortricidae). Journal of Economic Entomology, 96, 592-598.

Jordan, A.M., Nash, T.A.M. \& Boyle, J.A. (1969) Pupal weight in relation to female age in Glossina austeni Newst. Bulletin of Entomological Research, 58, 549-552.

Kabayo, J.P. \& Langley, P.A. (1985) The nutritional importance of dietary blood components for reproduction in the tsetse fly, Glossina morsitans. Journal of Insect Physiology, 31, 619-624.

Kaitala, A. (1991) Phenotypic plasticity in reproductive behaviour of waterstriders: Trade-offs between reproduction and longevity during food stress. Functional Ecology, 5, 12.

Kirkwood, T. (1977) Evolution of ageing. Nature, 270, 301-303.

Kyneb, A. \& Toft, S. (2006) Effects of maternal diet quality on offspring performance in the rove beetle Tachyporus hypnorum. Ecological Entomology, 31, 322-330.

Langley, P. A. (1977) Physiology of tsetse flies (Glossina spp.) (Diptera: Glossinidae): a review. Bulletin of Entomological Research, 67, 523.

Langley, P. \& Clutton-Brock, T. (1998) Does reproductive investment change with age in tsetse flies, Glossina morsitans morsitans (Diptera: Glossinidae)? Functional Ecology, 12, 866-870.

Mangel, M. \& Clark, C. (1988) Dynamic modelling in behavioral ecology. Princeton, NJ: Princeton University Press.

Mautz, B.S., Rode, N.O., Bonduriansky, R. \& Rundle, H.D. (2019) Comparing ageing and the effects of diet supplementation in wild vs. captive antler flies, Protopiophila litigata. Journal of Animal Ecology, 88, 1913-1924.

McIntyre, G.S. \& Gooding, R.H. (1998) Effect of maternal age on offspring quality in tsetse (Diptera: Glossinidae). Journal of Medical Entomology, 35, 210-215.

McNamara, J.M., Houston, A.I., Barta, Z., Scheuerlein, A. \& Fromhage, L. (2009) Deterioration, death and the evolution of reproductive restraint in late life. Proceedings of the Royal Society B-Biological Sciences, 276, 4061-4066.

Medawar, P. (1952) An Unsolved Problem of Biology. H. K: Lewis.

Monaghan, P., Maklakov, A.A. \& Metcalfe, N.B. (2020) Intergenerational transfer of ageing: Parental age and offspring lifespan. Trends in Ecology \& Evolution, 35, 927-937.

Moorad, J.A. \& Nussey, D.H. (2016) Evolution of maternal effect senescence. Proceedings of the National Academy of Sciences, 113, 362-367.

Mousseau, T.A. \& Dingle, H. (1991) Maternal effects in insect life histories. Annual Review of Entomology, 36, 511-534.

Nakagawa, S. \& Schielzeth, H. (2013) A general and simple method for obtaining R2 from generalized linear mixed-effects models. Methods in Ecology and Evolution, 4, 133-142.

Nussey, D., Froy, H., Lemaitre, J., Gaillard, J. \& Austad, S. (2013) Senescence in natural populations of animals: widespread evidence and its implications for bio-gerontology. Ageing Research Reviews, 23, 1-7.

Phelps, R. (1973) The effect of temperature on fat consumption during the puparial stages of Glossina morsitans morsitans Westw. (Dipt., Glossinidae) under laboratory conditions, and its implication in the field. Bulletin of Entomological Research, 62, 423.

Phelps, R. \& Burrows, P. (1969) Puparial duration in Glossina morsitans orientalis under conditions of constant temperature. Entomologia Experimentalis et Applicata, 12, 33-43.

Pinheiro, J., Bates, D., \& DebRoy, S. D. S. (2018). nlme: linear and nonlinear mixed effects models.
R Core Team (2014) R: A language and environment for statistical computing.

Reinhardt, K., Naylor, R.A. \& Siva-Jothy, M.T. (2009) Ejaculate components delay reproductive senescence while elevating female reproductive rate in an insect. Proceedings of the National Academy of Sciences, 106, 21743-21747.

Rodríguez-Muñoz, R., Boonekamp, J.J., Fisher, D., Hopwood, P. \& Tregenza, T. (2019) Slower senescence in a wild insect population in years with a more female-biased sex ratio. Proceedings of the Royal Society B: Biological Sciences, 286(1900), 20190286. https:// doi.org/10.1098/rspb.2019.0286.

Sharp, S.P. \& Clutton-Brock, T.H. (2010) Reproductive senescence in a cooperatively breeding mammal. Journal of Animal Ecology, 79, 176-183.

Stearns, S. (1992) The Evolution of Life Histories. Oxford, UK: Oxford University Press.

Tatar, M. \& Carey, J.R. (1995) Nutrition mediates reproductive tradeoffs with age-specific mortality in the beetle Callosobruchus maculatus. Ecology, 76, 2066-2073.

Unnithan, G.C. \& Paye, S.O. (1991) Mating, longevity, fecundity, and egg fertility of Chilo partellus (Lepidoptera: Pyralidae): Effects of delayed or successive matings and their relevance to pheromonal control methods. Environmental Entomology, 20, 150-155.

Van De Pol, M. \& Verhulst, S. (2006) Age-dependent traits: A new statistical model to separate within- and between-individual effects. American Naturalist, 167, 766-773.

Velando, A., Drummond, H. \& Torres, R. (2006) Senescent birds redouble reproductive effort when ill: Confirmation of the terminal investment hypothesis. Proceedings of the Royal Society B-Biological Sciences, 273, 1443-1448.

Wen, L., Zhang, Z., Zhang, S., Liu, F., Jiao, X. \& Li, D. (2020) Influence of maternal diet on offspring survivorship, growth, and reproduction in a sheetweb spider. Biology Open, 9(11), bio056846. https://doi.org/10.1242/bio.056846.

Williams, G. (1957) Pleiotropy, natural selection and the evolution of senescence. Evolution (N. Y), 11, 398-411.

Wood, S. (2017) Generalized additive models: an introduction with $R$, 2nd edn. London, UK: Chapman and Hall/ CRC.

Young, A.J., Carlson, A.A., Monfort, S.L., Russell, A.F., Bennett, N.C. \& Clutton-Brock, T. (2006) Stress and the suppression of subordinate reproduction in cooperatively breeding meerkats. Proceedings of the National Academy of Sciences, 103, 12005-12010.

Zajitschek, F., Hunt, J., Jennions, M.D., Hall, M.D. \& Brooks, R.C, (2009) Effects of juvenile and adult diet on ageing and reproductive effort of male and female black field crickets, Teleogryllus commodus. Functional Ecology, 23, 602-611.

Zajitschek, F., Zajitschek, S. \& Bonduriansky, R. (2019) Senescence in wild insects: Key questions and challenges. Functional Ecology, $26-37$.

\section{SUPPORTING INFORMATION}

Additional supporting information may be found online in the Supporting Information section.

How to cite this article: Lord, J.S., Leyland, R., Haines, L.R., Barreaux, A.M.G., Bonsall, M.B., Torr, S.J.\&et al (2021) Effects of maternal age and stress on offspring quality in a viviparous fly. Ecology Letters, 24, 2113-2122. https://doi.org/10.1111/ele.13839 